\title{
Controllable Assembly of Hydrophobic Superparamagnetic Iron Oxide Nanoparticle with mPEG-PLA Copolymer and Its Effect on MR Transverse Relaxation Rate
}

\author{
Xuan Xie and Chunfu Zhang \\ Med-X Research Institute, Shanghai Jiao Tong University, Shanghai 200030, China \\ Correspondence should be addressed to Chunfu Zhang, cfzhang@sjtu.edu.cn
}

Received 2 May 2010; Revised 14 July 2010; Accepted 15 August 2010

Academic Editor: William W. Yu

Copyright (C) 2011 X. Xie and C. Zhang. This is an open access article distributed under the Creative Commons Attribution License, which permits unrestricted use, distribution, and reproduction in any medium, provided the original work is properly cited.

\begin{abstract}
Assembly of individual superparamagnetic iron oxide nanoparticles (SPION) into cluster is an effective way to prepare MRI contrast agent with high relaxivity. In this study, we fabricated SPION clusters with different sizes and configurations by assembly of amphiphilic mPEG-PLA copolymer with hydrophobic SPION in aqueous solution. The evolution of cluster size and configuration with the amount of copolymer and the effect of cluster size on the transverse relaxivity was studied. T2 relaxation rates of clusters with different sizes at iron concentration of $0.1 \mathrm{mM}$ were compared with the theoretical predictions. We found that the relative amount of copolymer/SPION was crucial for the formation of SPION cluster. The transverse relaxivity of the condense SPION clusters (CSC) was size-dependent. The experimentally measured T2 relaxation rates of the clusters were lower than the theoretical predictions. In motional average regime (MAR) region, $T 2$ relaxation rates were more consistent with the theoretical values when transmission electron microscope (TEM) evaluated size was used. Therefore, for fabrication of SPION clusters with assembly of mPEG-PLA and hydrophobic SPION, delicate balance between the amount of copolymer and SPION should be pursued, and for comparison of experimental $T 2$ relaxation rate with theoretical predictions, TEM evaluated size was more suitable.
\end{abstract}

\section{Introduction}

Magnetic resonance imaging (MRI) is among the best noninvasive methodologies today in clinical medicine for assessing anatomy and function of tissues. The MRI technique offers several advantages such as excellent temporal and spatial resolution, the lack of exposure to radiation, rapid in vivo acquisition of images, and long effective imaging window [1]. These strengths make it a highly desirable modality for MR molecular imaging. However, due to its relatively lower sensitivity towards traditional small molecule contrast agents, such as Gd-DTPA, its success in this research field suffers.

Superparamagnetic iron oxide nanoparticle (SPION), because of its biocompatibility and much higher sensitivity than gadolinium contrast agent, is preferable for MR contrast enhancement. Currently, SPION coated with dextran and its derivatives have been used in clinical routine and also extensively explored for MR molecular imaging [2, 3]. However, for detecting targets with lower expression level, contrast agents with much higher sensitivity are superior. Ultrahigh sensitive MnMEIO probe had been demonstrated not only to detect the expression but also to differentiate the expression level of HER2/Neu on tumor cells [4].

Increasing SPION size can increase its transverse relaxivity [5]. However, the large particles (at a size approximately larger than $15 \mathrm{~nm}$ ) are not superparamagnetic [6] and easily aggregate in solution. Another way to increase T2 relaxivity, while keeping the superparamagnetic characteristics, is clustering individual SPION into clusters. Using polyelectrolyteneutral block copolymers and by electrostatic adsorption and charge compensation between oppositely charged species, Berret et al. [7] fabricated maghemite nanocluster and tuned the size of aggregates in the range of 70-150 nm with aggregation numbers (number of nanoparticles per aggregate) from tens to hundreds. It was found that the transverse 
relaxivity, $r_{2}$, was noticeably increased with the size of the magnetic clusters. Ai et al. had also obtained similar result for magnetic nanoparticles encapsulated into the hydrophobic cores of 20-100 $\mathrm{nm}$ polymeric micelles [8]. These studies indicated that clustering magnetic nanoparticles resulted in enhanced transverse relaxivity. In recent years, different strategies for fabrication of superparamagnetic magnetic nanoclusters had been put forward [9-11].

mPEG-PLA is an amphiphilic copolymer and ideal for fabrication of SPION cluster for MR molecular imaging in vivo. First, both PEG and PLA are FDA allowed. Second, the properties of PLA, such as crystallinity, tensile strength and hydrophobicity, can be easily modulated, thus the loading capacity for hydrophobic SPION can be tuned [12]. In addition, PEG is nonimmunogenic and highly hydrophilic. Surface coating with PEG has been demonstrated to extend nanoparticle circulation time in vivo, leading to better targeting behavior [13]. mPEG-PLA micelles have been shown to be able to load hydrophobic SPION and doxorubicin simultaneously and hold great promise for MR molecular imaging [10].

So, in this study, we fabricated SPION cluster with high MR sensitivity by assembly of hydrophobic SPION with amphiphilic mPEG-PLA copolymer. The relaxation rates of the clusters with different sizes and configuration were studied and compared with theoretical predictions. The condense SPION cluster (CSC) contrast agent may find its uses in MR molecular imaging of targets on tumor angiogenic vessels, such as, $\alpha_{v} \beta_{3}$, VEGFR, and vascular cell adhesion molecule (VCAM).

\section{Experimental}

2.1. Chemicals. Poly(ethylene glycol) methyl ether (mPEG, Mw 5000), D,L-lactide, Sn(II) octoate, Fe(acac) 3 , benzyl ether, oleylamine(70\%), and 1,2-hexadecanediol(90\%) were purchased from Sigma (St. Louis, MO). Other chemical reagents were obtained from Sinopharm Chemical Reagent Co. (China). Toluene was dried by refluxing over sodium under dry argon. All aqueous solutions were prepared with water from a Milli-Q water purification system.

2.2. Synthesis of mPEG-PLA Copolymer. mPEG-PLA was synthesized by ring opening polymerization of D,L-lactide at $110^{\circ} \mathrm{C}$ [10]. In detail, $1 \mathrm{~g}$ poly(ethylene glycol) methyl ether (mPEG, Mw 5000) was added into two-necked round flask, heated at $80^{\circ} \mathrm{C}$ under vacuum for $1 \mathrm{~h}$, and subsequently cooled down to the ambient temperature. D, L-Lactide (1 g) was then added into the flask, and vacuumed overnight. Subsequently, freshly distilled toluene $(10 \mathrm{~mL})$ was injected into the flask and the polymerization was initiated by adding tin(II) 2-ethylhexanonate $(60 \mu \mathrm{L})$ as catalyst at $110^{\circ} \mathrm{C}$ with nitrogen flushing. After the reaction was performed for $4 \mathrm{~h}$, the mixture was allowed to cool down to the ambient temperature, and toluene was removed by rotary evaporator. The final product was purified with THF three times.

2.3. Synthesis of Hydrophobic SPION. The hydrophobic SPIONs were synthesized following the published procedure
[11] with little modification. Briefly, $\mathrm{Fe}(\mathrm{acac})_{3}(1 \mathrm{mmol})$, 1,2-hexadecanediol ( $5 \mathrm{mmol})$, oleic acid ( $3 \mathrm{mmol})$, oleylamine $(3 \mathrm{mmol})$, and benzyl ether $(10 \mathrm{~mL})$ were mixed into a three-necked flask and magnetically stirred under a flow of nitrogen. The mixture was heated to $200^{\circ} \mathrm{C}$ for $2 \mathrm{~h}$ under a blanket of nitrogen and refluxed at $300^{\circ} \mathrm{C}$ for $1 \mathrm{~h}$. After cooling to the ambient temperature, the solution was treated with ethanol and centrifuged to yield a dark-brown precipitate. The precipitate was washed with ethanol three times and finally dispersed in toluene.

2.4. Assembly of mPEG-PLA with Hydrophobic SPION. For assembly of mPEG-PLA with hydrophobic SPION, the hydrophobic SPION in toluene was first dried with nitrogen flow and mixed with mPEG-PLA (in $1 \mathrm{~mL}$ THF solution). Then, the mixture was quickly injected into water $(5 \mathrm{~mL})$ with vigorous stirring. THF was removed by evaporation at room temperature overnight. By varying the amount of SPION $(0.67,1.33 \mathrm{mg})$ or mPEG-PLA $(0.1-0.5 \mathrm{mg})$ used, different size and configuration of magnetite clusters were able to be prepared.

\subsection{Characterizations}

2.5.1. ${ }^{1}$ H Nuclear Magnetic Resonance. The polymerization of mPEG with D, L-Lactide monomers was characterized with nuclear magnetic resonance (Bruker Avance $500 \mathrm{MHz}$ NMR, USA). The degree of polymerization was calculated by comparing integral intensity of characteristic resonance of PLA at $5.2 \mathrm{ppm}\left(-\mathrm{C}(=\mathrm{O})-\mathrm{CH}\left(-\mathrm{CH}_{3}-\right)\right)$ and $\mathrm{mPEG}$ resonance at $3.64 \mathrm{ppm}\left(-\mathrm{OCH}_{2} \mathrm{CH}_{2-}\right)$ in the ${ }^{1} \mathrm{H}$ spectra.

2.5.2. Transmission Electron Microscope (TEM) . The morphology, size, and size distribution of the hydrophobic SPION and SPION clusters were characterized by TEM (JEOL 2100F, Japan). The particle suspension was directly deposited onto a carbon-coated copper grid and air-dried at room temperature. The particle size and size distribution were calculated using an image analysis program by measuring the diameter of at least 300 particles.

2.5.3. Hydrodynamic Size. The hydrodynamic size of the clusters was measured by dynamic light scattering (DLS) using a Malvern Autosizer 4700/PCS100 spectrometer equipped with an Ar ion laser operating at $488 \mathrm{~nm}$. Triple measurements were performed, and the number- and volume-weighted mean size were taken.

2.5.4. T2 Relaxometry. The relaxometry was performed using a $1.4 \mathrm{~T}$ minispec mq60 NMR Analyzer (Bruker, Germany) following the reported method [14]. The micelles were suspended at the iron concentrations between 0.001 and $10 \mathrm{mM}$. For MR measurements, $0.3 \mathrm{~mL}$ micelle dilutions were filled into the test tubes, and $T 2$ relaxation times were measured at $310 \mathrm{~K}$ using a standard Carr-Purcell-MeiboomGill pulse sequence with echo time $\tau_{\mathrm{CP}}=0.5 \mathrm{~ms}$. The $T 2$ relaxivities were determined by a linear fit of the inverse relaxation times as a function of the iron concentrations. 


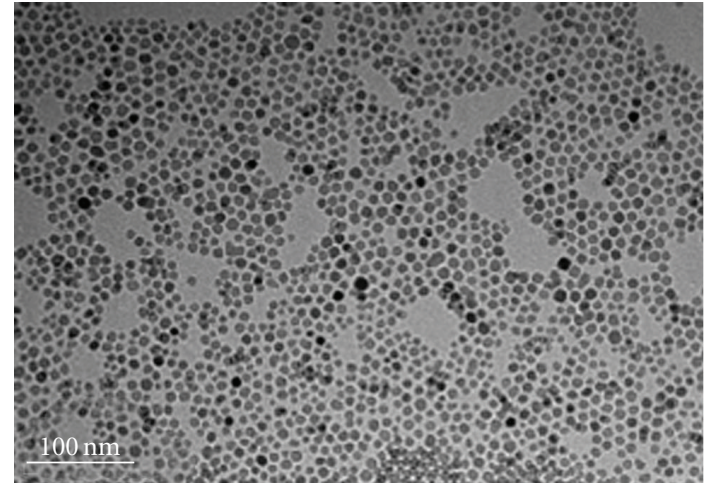

(a)

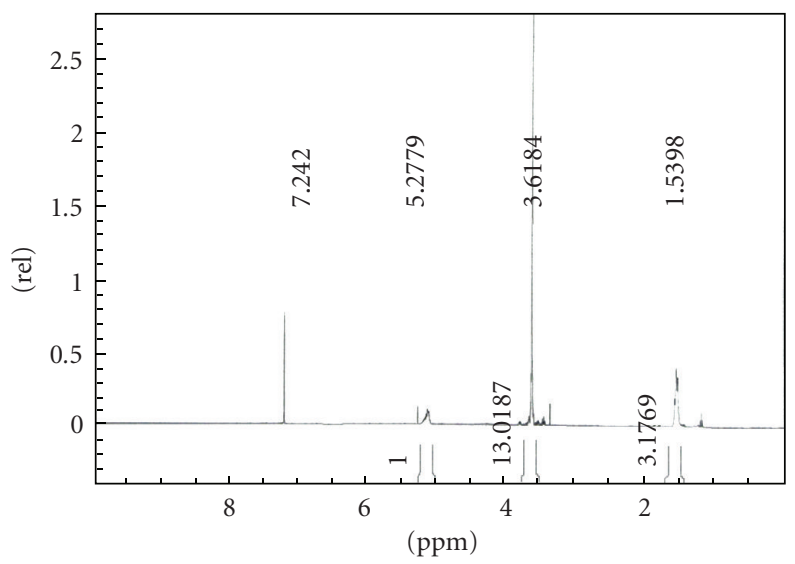

(b)

FIGURE 1: (a) TEM image of hydrophobic superparamagnetic iron oxide nanoparticles. The size of the particles is about $6 \mathrm{~nm}$. (b) $1 \mathrm{H}$ NMR spectrum of PEG (5 K)-PLA(2.5 K).

2.5.5. Vibrating Sample Magnetometer (VSM). Magnetic properties of hydrophobic SPION and SPION clusters were characterized in a vibrating sample magnetometer (LakeShore7300, USA). The tested samples were lyophilized and dried at $80^{\circ} \mathrm{C}$ under vacuum prior to analysis. The magnetization $\left(M\right.$, emu $\left.\mathrm{g}^{-1}\right)$ of the samples was measured as a function of the magnetic field $(\mathrm{H}, \mathrm{Oe})$ at $298 \mathrm{~K}$.

2.5.6. Thermogravimetric Analysis (TGA). TGA analysis for hydrophobic SPION and SPION clusters was performed with a NETZSCH TG 209 F1 iris instrument (NETZSCH, Germany) from room temperature to $1000^{\circ} \mathrm{C}$ with a heating rate of $10^{\circ} \mathrm{C} \mathrm{min}^{-1}$ in a nitrogen flow $\left(20 \mathrm{~mL} \mathrm{~min}^{-1}\right)$. The initial weight of sample was $10 \mathrm{mg}$. The weight fraction (wt\%) of organic component (mPEG-PLA) and SPION in the clusters were deduced from the TGA curve.

\section{Results and Discussion}

3.1. Synthesis and Characterizations of SPION and SPION Clusters. By thermal decomposition of iron precursors in the organic solvent in the presence of surfactants, hydrophobic SPION with tunable sizes can be synthesized [11]. In this

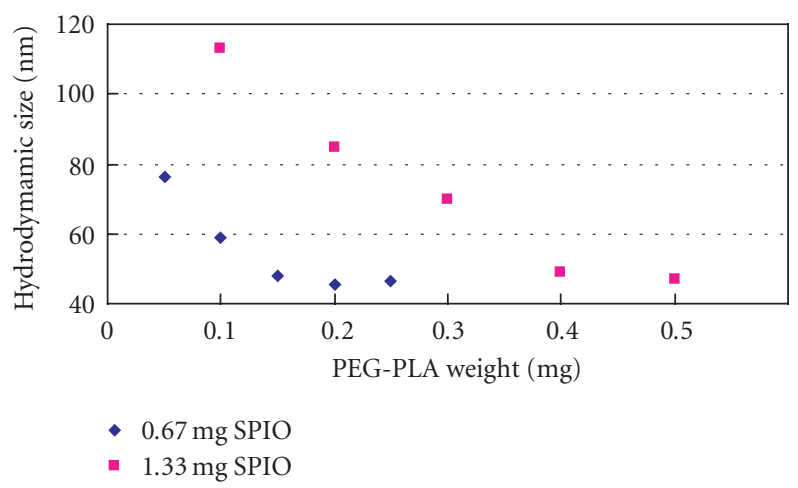

FIGURE 2: Evolution of hydrodynamic size (number weighted) of SPION clusters fabricated with different amounts of hydrophobic SPION or amphiphilic copolymer.

study, the size of SPION synthesized was about $6 \mathrm{~nm}$, and it was coated with oleic acid and dispersed in toluene very well (Figure 1(a)) [15].

The polymerization of D,L-lactide monomers with mPEG induced by hydroxyl group was manifested by ${ }^{1} \mathrm{H}$ NMR spectrum. The characteristic peaks occurring at $5.2 \mathrm{ppm}$ and $3.64 \mathrm{ppm}$ in the ${ }^{1} \mathrm{H}$ NMR spectrum were the resonance of methine protons in PLA $\left(-\mathrm{C}(=\mathrm{O})-\mathrm{CH}\left(-\mathrm{CH}_{3}-\right)\right)$ and methylene protons in PEG (-OCH $\left.\mathrm{CH}_{2}-\right)$, respectively. By varying the amount of catalyst, mPEG-PLA copolymers with different molecular weights (Mw: 7100, 7500, 8400, and 9340) were synthesized. The representative ${ }^{1} \mathrm{H}$ NMR spectrum of mPEG-PLA with molecular weight of 7500 was shown in Figure 1(b).

For fabrication of SPION clusters, $0.67 \mathrm{mg}$ hydrophobic SPION was first used to assemble with different amounts of mPEG-PLA. Increasing the amount of copolymer (Mw 7500) from $0.05 \mathrm{mg}$ to $0.2 \mathrm{mg}$ led to the decrease in the assembly size (number-weighted size, Figure 2). When the copolymer mass was more than $0.2 \mathrm{mg}$, the size of the cluster would not change significantly and maintained at about $45 \mathrm{~nm}$. Morphologically, condense SPION cluster (CSC, Figure 3(a)) or blank mPEG-PLA micelle with SPION adhesion on the surface (referred to "loosen SPION cluster," LSC) (Figure 3(b)) was observed during the change of cluster sizes. The evolution of cluster size and configuration with polymer amount were similar when double amount of SPION $(1.33 \mathrm{mg})$ was utilized. However, a rise in SPION amount resulted in dramatical size increase (Figure 2).

The assembly of mPEG-PLA with hydrophobic SPION in aqueous solution is the result of strong absorption of SPION with the hydrophobic segment (PLA) of the amphiphilic polymer. Upon mPEG-PLA, SPION, and THF mixture is added into aqueous solution, the hydrophobic PLA chain is preferred to be inserted into the space between the oleic acids on SPION surface with hydrophilic PEG chain extending outside. With increase in mPEG-PLA amount in the aqueous solution, more polymer will be available for individual SPION and less SPION would be included in one cluster, which may decrease the cluster size. However, single SPION coated with mPEG-PLA was not found. 


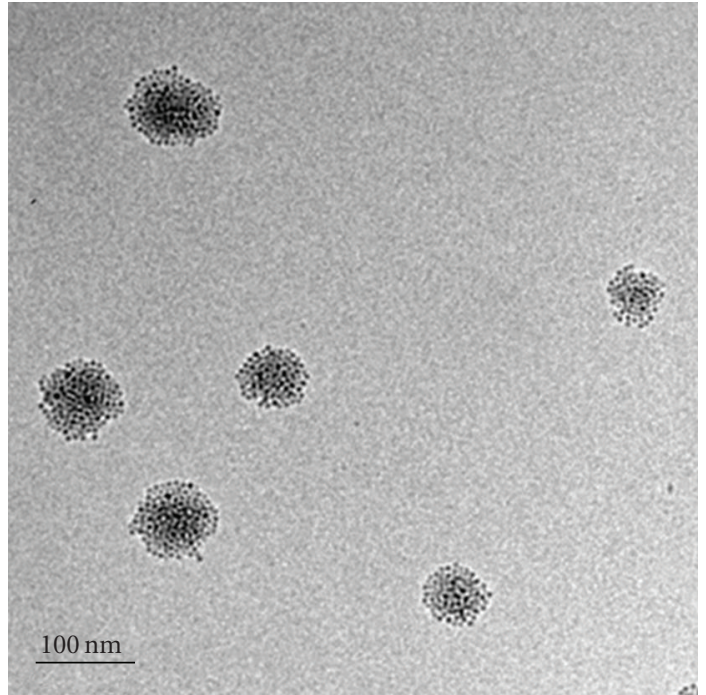

(a)

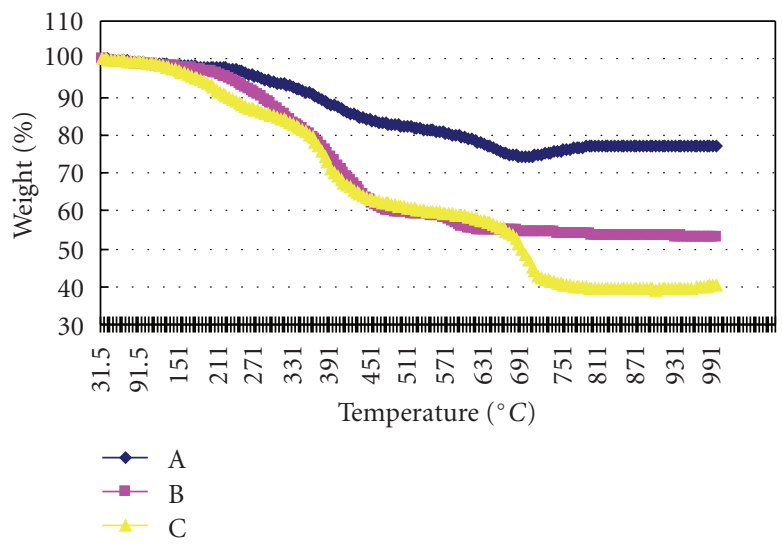

(c)

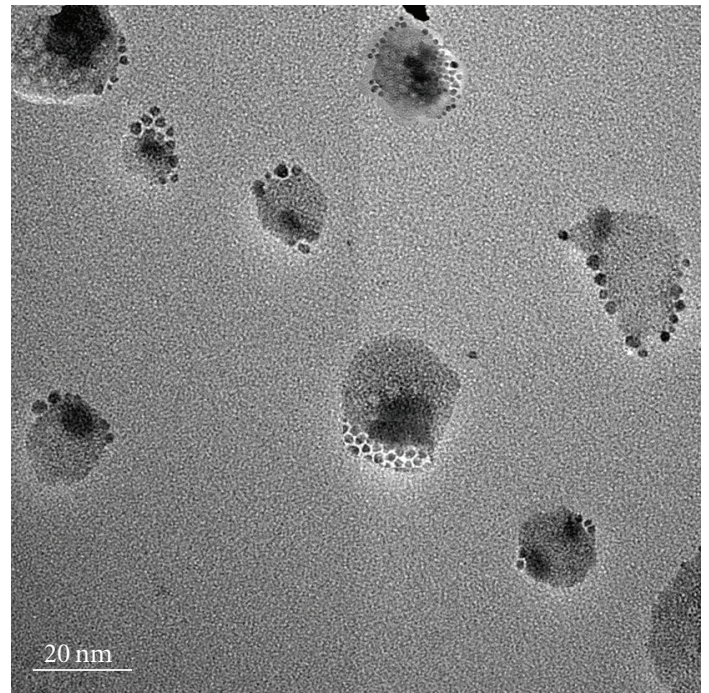

(b)

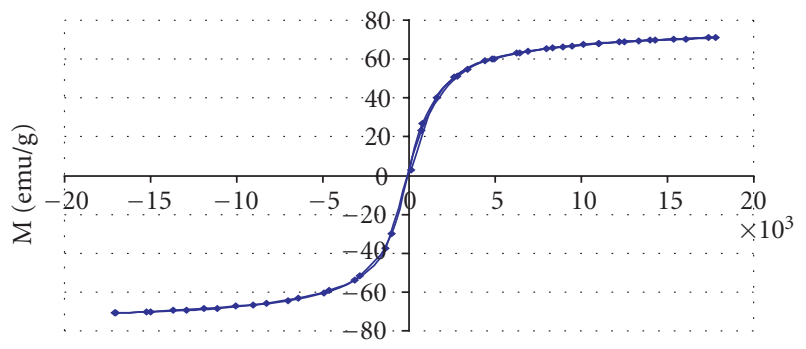

$\mathrm{H}(\mathrm{O}$ e)

(d)

FIgURE 3: Characterizations of SPION clusters. (a) and (b) Representative TEM images of condense SPION clusters (CSC) and loosen SPION cluster (LSC), respectively. (c) TGA curve of hydrophobic SPION (A), condense SPION clusters (B) and loosen SPION cluster (C). (d) Magnetization plot of SPION as a function of the applied field at $298 \mathrm{~K}$.

For fabrication of condense SPION cluster (CSC), Mw of mPEG-PLA did not affect the size of cluster significantly. As shown in Figure 2, in the case of assembly of $0.1 \mathrm{mg}$ copolymer with $0.67 \mathrm{mg}$ SPION with copolymer Mw of 7500, the size of cluster was $58.3 \mathrm{~nm}$. When copolymers of different Mw (7100, 8400, and 9340) were used for assembly with identical mass of copolymer $(0.1 \mathrm{mg})$ and SPION $(0.67 \mathrm{mg})$, the sizes of the clusters were $60.6 \mathrm{~nm}, 58.1 \mathrm{~nm}$, and $63.2 \mathrm{~nm}$, respectively.

TGA curves of hydrophobic SPION, CSC, or LSC showed that the first step of thermal decomposition was around $100^{\circ} \mathrm{C}$ and the major decomposition occurred in the temperature range at $200-800^{\circ} \mathrm{C}$. Less weight loss occurred at temperatures above $800^{\circ} \mathrm{C}$ (Figure 3(c)). The initial weight loss was due to removal of surface adsorbed water (hydrophobic SPION: $2.12 \%$, CSC: $3.33 \%$, and LSC: $6.87 \%$ ). The weight loss between $200-800^{\circ} \mathrm{C}$ was attributed to the thermal degradation of organic components on the SPION surface or in the clusters $23.56 \%$ for hydrophobic SPION,
$42.92 \%$ for CSC, and $55.55 \%$ for LSC). The contents for residues (SPION) were $74.32 \%$ for hydrophobic SPION, $53.74 \%$ for CSC, and $37.58 \%$ for LSC, respectively.

Magnetic properties of hydrophobic SPION, CSC and LSC were studied by using a vibration sample magnetometer (VSM). According to the SPION content deduced from TGA data, the saturation magnetization (Ms) value of SPION was found to be $66.73 \mathrm{emu} \mathrm{g}^{-1}$ (Figure 3(d)). The shape of the hysteresis curve was normal and tight with no hysteresis losses, which was expected as the behavior of superparamagnet [16].

T2 relaxation rate $(1 / T 2)$ of SPION cluster was fitted as a function of iron concentrations (Figure 4(a)). For condense SPION cluster (CSC), T2 relaxivities were 234, 363 , and $413 \mathrm{~s}^{-1} \mathrm{mM}^{-1}$ at the sizes (number-weighted) of 49,59 , and $76 \mathrm{~nm}$, respectively. When the size of the cluster reached $113 \mathrm{~nm}$, the $T 2$ relaxivity was as high as $512 \mathrm{~s}^{-1} \mathrm{mM}^{-1}$. These findings were consistent with previous reports that clustering magnetic nanoparticles resulted in 


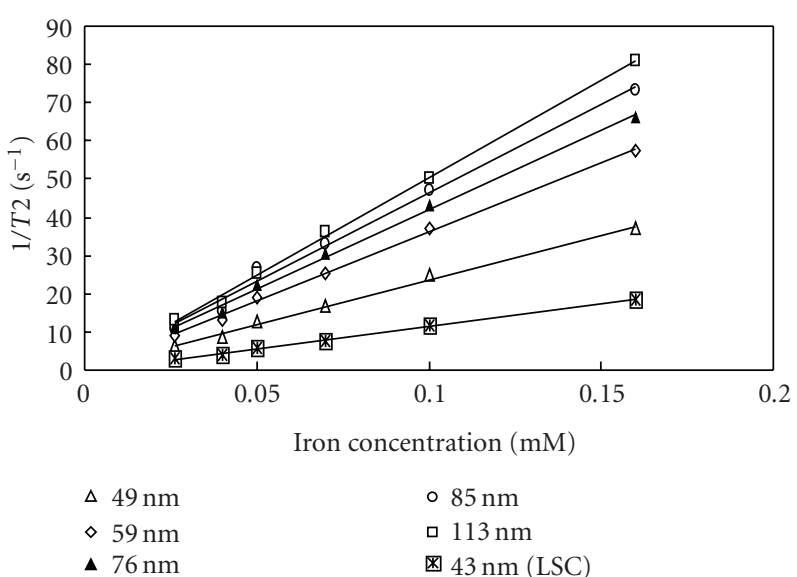

(a)

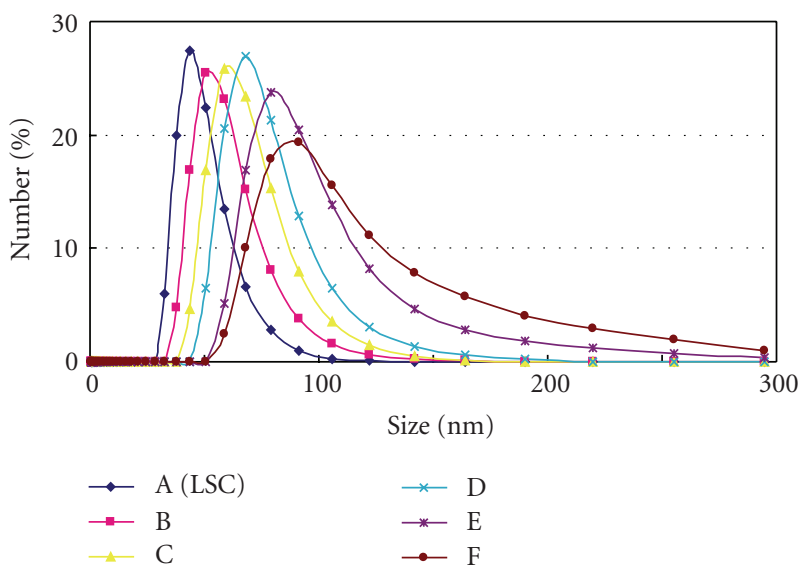

(c)

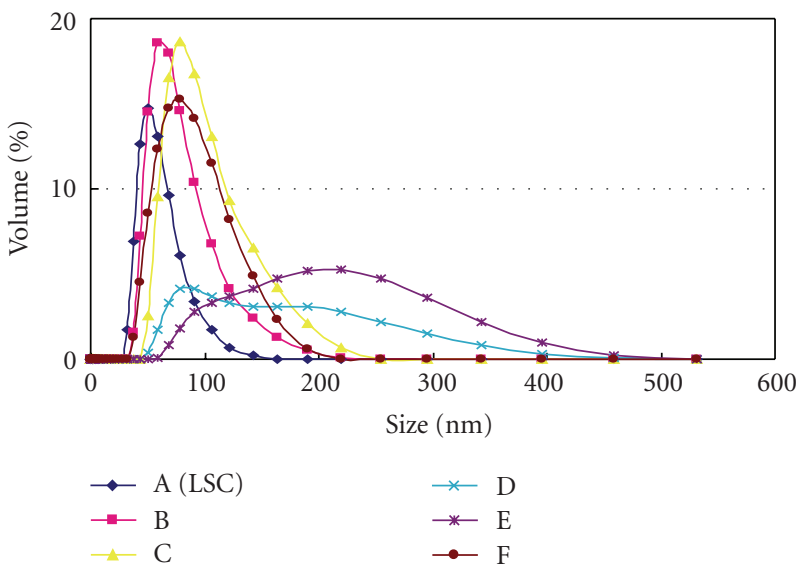

(b)

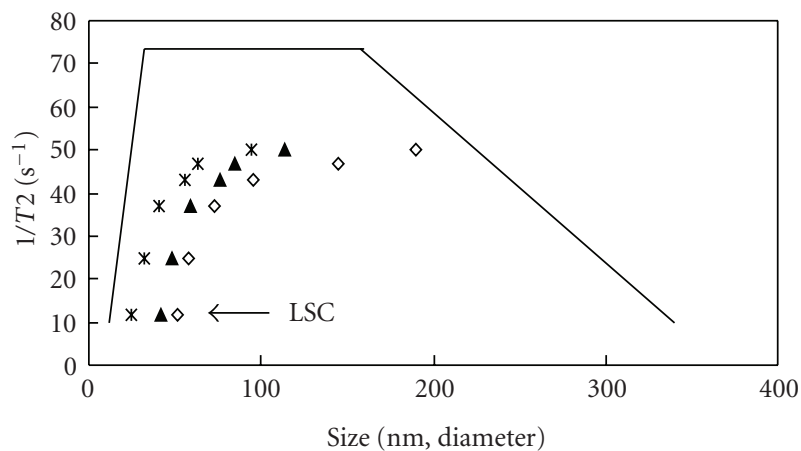

\ Number-weight size

$\diamond$ Volume-weight size

* TEM-evaluated size

(d)

FIGURE 4: MR relaxation property of SPION clusters, these size distributions and comparison of the relaxation rates with theoretical predictions (d). (a) Relaxation rates of SPION clusters as functions of iron concentrations. (b) Size distribution of SPION clusters evaluated with volume-weighted method. (A) loosen SPION clusters (LSC): $58 \mathrm{~nm}$. (B)-(F), condense SPION clusters (CSC): $73 \mathrm{~nm}, 95 \mathrm{~nm}, 97 \mathrm{~nm}$, $144 \mathrm{~nm}$ and $199 \mathrm{~nm}$, respectively. (c) Size distribution of SPION clusters evaluated with number-weighted method. (A) loosen SPION clusters (LSC): $43 \mathrm{~nm}$. (B)-(F), condense SPION clusters (CSC): $48 \mathrm{~nm}, 59 \mathrm{~nm}, 76 \mathrm{~nm}, 85 \mathrm{~nm}$ and $113 \mathrm{~nm}$, respectively. (d) Comparison of relaxation rates of SPION clusters with theoretical predictions with sizes evaluated with TEM or DLS (number-weighted and volumeweighted). The straight lines are fair approximations of the theoretical predictions (MAR, SD and ELR). Loosen SPION clusters (LSC) demonstrate the smallest sizes and relaxation rate.

enhanced transverse relaxivity $[7,8]$. Whereas, for loosen SPION cluster (LSC), the T2 relaxivity was comparatively low $\left(43 \mathrm{~nm}, r_{2}=116.94 \mathrm{~s}^{-1} \mathrm{mM}^{-1}\right)$.

\subsection{Comparison of the Experimental T2 Relaxation Rates} with Theoretical Predictions. In order to compare the experimental $T 2$ relaxation rates with theoretical predictions, the T2 relaxation rates versus volume-weighted (Figure 4(b)) and number-weighted (Figure 4(c)) hydrodynamic sizes of SPION clusters were plotted at $0.1 \mathrm{mM}$ iron concentration. The $T 2$ relaxation rate of water in magnetic particle suspension is predicted by different regimes according to the size of the particle [17]. When particles are small, the T2 relaxation rate will be dominated by water molecular motions (motional average regime, MAR). $1 / T 2$ is proportional to $\tau_{D}$

$$
\frac{1}{T 2}=\frac{16 f_{v} \Delta \omega^{2} \tau D}{45}
$$

where $f_{v}$ is the volume fraction of magnetic particles, $\Delta \omega$ is the difference in angular frequency between the local field experienced by a proton at the equatorial line of the cluster surface and in the bulk, and $\tau_{D}$ is the translational diffusion time around the cluster, in which

$$
\begin{gathered}
\Delta \omega=\frac{\gamma \mu_{0} M}{3}, \\
\tau_{D}=\frac{R^{2}}{D} .
\end{gathered}
$$

$\gamma$ is the proton gyromagnetic ratio $(42.58 \mathrm{MHz} / \mathrm{T}) ; \mu_{0}$ the vacuum magnetic permeability $\left(4 \pi \times 10^{-7} \mathrm{Tm} / \mathrm{A}\right) ; M$ the particle magnetization.; $R$ the particle radius; $D_{w}$ the selfdiffusion coefficient of pure water at $310 \mathrm{~K}\left(3 \times 10^{9} \mathrm{~m}^{2} \mathrm{~s}^{-1}\right)$ [18]. 
When particle size increases to a certain size, the $T 2$ relaxation rate reaches its maximum and is governed by static dephasing regime (SDR):

$$
\frac{1}{T 2}=\frac{2 \pi \sqrt{ } 3 f_{v} \Delta \omega}{9} .
$$

Above this size, it plateaus. Further size increase would result in decrease of $T 2$ relaxation rate, which can be predicted by echo-limited regime (ELR). In this regime, $T 2$ relaxation rate is proportional to $1 / \tau_{D}$ and is related to $\tau_{\mathrm{CP}}$, with

$$
\frac{1}{T 2}=\frac{1.8 f_{v}\left(\Delta \omega \tau_{\mathrm{CP}}\right)^{1 / 3}\left(1.52+f_{v} \Delta \omega \tau_{\mathrm{CP}}\right)^{5 / 3}}{\tau_{D}} .
$$

The intersections of SDR with MAR (left edge of SDR, $\tau_{D 1}$ ) and ELR ( right edge of SDR, $\tau_{D 2}$ ) are, respectively, at

$$
\begin{gathered}
\tau_{D 1}=\frac{5 \pi \sqrt{ } 3}{8 \Delta \omega} \\
\tau_{D 2}=\left(\frac{1.49}{\Delta \omega}\right)\left(\Delta \omega \tau_{\mathrm{CP}}\right)^{1 / 3}\left(1.52+f_{v} \Delta \omega \tau_{\mathrm{CP}}\right)^{5 / 3}
\end{gathered}
$$

[18].

Using the measured magnetization of SPION $(66.73 \mathrm{emu} / \mathrm{g})$, the theoretical $1 / T 2$ versus size of the particle (diameter) can be calculated by (1), (2), (4), and (5) with $\tau_{\mathrm{CP}}=0.5 \mathrm{~ms}$ and $[\mathrm{Fe}]=0.1 \mathrm{mM}\left(f_{v}=1.52 \times 10^{-5}\right.$ [Fe] [17]). The result was plotted in Figure $4(\mathrm{~d})$ as a line consisting of three straight segments. The experimental $1 / T 2$ data points were also shown in the figure versus number-weighted or volume-weighted hydrodynamic size.

According to (6) and (7), the calculated left edge and right edge of SDR are about $30 \mathrm{~nm}$ and $160 \mathrm{~nm}$, respectively, which means that SDR is satisfied in this size range and T2 relaxation rate reaches its maximum $\left(73.48 \mathrm{~s}^{-1}\right)$ and keeps unchanged. In our case, the sizes of SPION cluster (number-weighted or volume-weighted) all fall in this size range. However, for CSC, T2 relaxation rates increase (from $25 \mathrm{~s}^{-1}$ to $43 \mathrm{~s}^{-1}$ ) with cluster size increase, expressing MAR behavior and reach its maximum at size about $85 \mathrm{~nm}$ for number-weighted size and $144 \mathrm{~nm}$ for volume-weighted size. Moreover, compared with theoretical prediction, the measured $T 2$ relaxation rate is much lower (for both MAR and SDR). This phenomena has also been observed for a styrene-acrylic acid copolymer-coated or dextran-coated iron oxide particles [18]. For LSC, the relaxation rate was also lower than that of theoretical prediction.

The possible explanation of the lower experimental value in MAR region may be that size evaluated by DLS with number-weighted or volume-weighted method was overestimated. When TEM-evaluated cluster size, which measures the size in "dry" state and may reflect the size more close to the "real" one, was applied, the measured relaxation rates were more closer to the theoretical line, which was consistent with the observation of micogel iron oxide particles [19]. However, in the SDR region, replacement with TEM size does not narrow the discrepancy between the experimental values and the theoretical prediction. Comparison of the experimental $T 2$ relaxation rate of a particular SPION formulation with that of theoretical prediction is complicated, which may involve the methods used for particle size evaluation $[20,21]$, magnetic property, surface coating, and even different formulation of the particles $[18,20]$. The low $T 2$ relaxation rate of the studied suspensions of iron-oxide particle clusters may be a general phenomena for all water suspensions of iron-oxide particles, and further experimental and theoretical studies are needed to confirm and understand it [18].

\section{Conclusion}

In this study, we fabricated magnetite nanoclusters with hydrophobic SPION (about $6 \mathrm{~nm}$ ) and mPEG-PLA amphiphilic copolymer and compared the $T 2$ relaxation rates with theoretical predictions. We found that the relative amount of mPEG-PLA and SPION for assembly played a crucial role in determining the size and configuration of the clusters. Condense SPION cluster or polymer sphere with surface SPION adhesion could be formed with different amounts of copolymer/SPION. For condense SPION clusters, T2 relaxivity of the cluster was size dependent. $T 2$ relaxation rates of the clusters were lower than those of theoretical predictions. Compared with DLS method, TEM-evaluated size was more suitable for the comparison of experimental $T 2$ relaxation rate with the theoretical prediction. In this regard, further experimental and theoretical studies are needed in the future to understand it.

\section{Acknowledgments}

This work was supported by research Grants from National Nature Science Foundation of China (30870682), Science and Technology Commission of Shanghai Municipality (08ZR1411100, 0852nm04600), Major State Basic Research Development Program of China (973 Program) (no. 2010CB834303), SRF for ROCS, SEM, and Research Fund for the Doctoral Program of Higher Education of China (20090073120004).

\section{References}

[1] P. Debbage and W. Jaschke, "Molecular imaging with nanoparticles: giant roles for dwarf actors," Histochemistry and Cell Biology, vol. 130, no. 5, pp. 845-875, 2008.

[2] S. Laurent, D. Forge, M. Port et al., "Magnetic iron oxide nanoparticles: synthesis, stabilization, vectorization, physicochemical characterizations and biological applications," Chemical Reviews, vol. 108, no. 6, pp. 2064-2110, 2008.

[3] C. Corot, P. Robert, J.-M. Idée, and M. Port, "Recent advances in iron oxide nanocrystal technology for medical imaging," Advanced Drug Delivery Reviews, vol. 58, no. 14, pp. 14711504, 2006.

[4] J.-H. Lee, Y.-M. Huh, Y.-W. Jun et al., "Artificially engineered magnetic nanoparticles for ultra-sensitive molecular imaging," Nature Medicine, vol. 13, no. 1, pp. 95-99, 2007.

[5] M. R. J. Carroll, R. C. Woodward, M. J. House et al., "Experimental validation of proton transverse relaxivity models for superparamagnetic nanoparticle MRI contrast agents," Nanotechnology, vol. 21, no. 3, Article ID 035103, 2010. 
[6] C.P. Bean and J. D. Livingston, "Superparamagnetism," Journal of Applied Physics, vol. 30, p. S120, 1959.

[7] J.-F. Berret, N. Schonbeck, F. Gazeau et al., "Controlled clustering of superparamagnetic nanoparticles using block copolymers: design of new contrast agents for magnetic resonance imaging," Journal of the American Chemical Society, vol. 128, no. 5, pp. 1755-1761, 2006.

[8] H. Ai, C. Flask, B. Weinberg et al., "Magnetite-loaded polymeric micelles as ultrasensitive magnetic-resonance probes," Advanced Materials, vol. 17, no. 16, pp. 1949-1952, 2005.

[9] G. Riess, "Micellization of block copolymers," Progress in Polymer Science, vol. 28, no. 7, pp. 1107-1170, 2003.

[10] N. Nasongkla, E. Bey, J. Ren et al., "Multifunctional polymeric micelles as cancer-targeted, MRI-ultrasensitive drug delivery systems," Nano Letters, vol. 6, no. 11, pp. 2427-2430, 2006.

[11] S. Sun, H. Zeng, D. B. Robinson et al., "Monodisperse $\mathrm{MFe}_{2} \mathrm{O}_{4}$ ( $\mathrm{M}=\mathrm{Fe}, \mathrm{Co}, \mathrm{Mn}$ ) Nanoparticles," Journal of the American Chemical Society, vol. 126, no. 1, pp. 273-279, 2004.

[12] Y. Li, X. R. Qi, Y. Maitani, and T. Nagai, "PEG-PLA diblock copolymer micelle-like nanoparticles as all-trans-retinoic acid carrier: in vitro and in vivo characterizations," Nanotechnology, vol. 20, no. 5, Article ID 055106, 2009.

[13] E. Blanco, C. W. Kessinger, B. D. Sumer, and J. Gao, "Multifunctional micellar nanomedicine for cancer therapy," Experimental Biology and Medicine, vol. 234, no. 2, pp. 123$131,2009$.

[14] N. Sun, D.-X. Chen, H.-C. Gu, and X.-L. Wang, "Experimental study on T2 relaxation time of protons in water suspensions of iron-oxide nanoparticles: waiting time dependence," Journal of Magnetism and Magnetic Materials, vol. 321, no. 18, pp. 29712975, 2009.

[15] F. Xu, D. Lei, and X. Du, "Modification of MR molecular imaging probes with cysteine- terminated peptides and their potential for in vivo tumour detection," Contrast Media and Molecular Imaging. In press.

[16] A. Bumb, M. W. Brechbiel, P. L. Choyke et al., "Synthesis and characterization of ultra-small superparamagnetic iron oxide nanoparticles thinly coated with silica," Nanotechnology, vol. 19, no. 33, Article ID 335601, 6 pages, 2008.

[17] A. Roch, Y. Gossuin, R. N. Muller, and P. Gillis, "Superparamagnetic colloid suspensions: water magnetic relaxation and clustering," Journal of Magnetism and Magnetic Materials, vol. 293, no. 1, pp. 532-539, 2005.

[18] D.-X. Chen, N. Sun, Z.-J. Huang, C.-M. Cheng, H. Xu, and H.C. $\mathrm{Gu}$, "Experimental study on T2 relaxation time of protons in water suspensions of iron-oxide nanoparticles: effects of polymer coating thickness and over-low 1/T2," Journal of Magnetism and Magnetic Materials, vol. 322, no. 5, pp. 548$556,2010$.

[19] E. S. M. Lee, B. Shuter, J. Chan et al., "The use of microgel iron oxide nanoparticles in studies of magnetic resonance relaxation and endothelial progenitor cell labelling," Biomaterials, vol. 31, no. 12, pp. 3296-3306, 2010.

[20] D.-X. Chen, N. Sun, and H.-C. Gu, "Size analysis of carboxydextran coated superparamagnetic iron oxide particles used as contrast agents of magnetic resonance imaging," Journal of Applied Physics, vol. 106, no. 6, Article ID 063906, 2009.

[21] D.-X. Chen, A. Sanchez, E. Taboada, A. Roig, N. Sun, and H.-C. Gu, "Size determination of superparamagnetic nanoparticles from magnetization curve," Journal of Applied Physics, vol. 105, no. 8, Article ID 083924, 2009. 

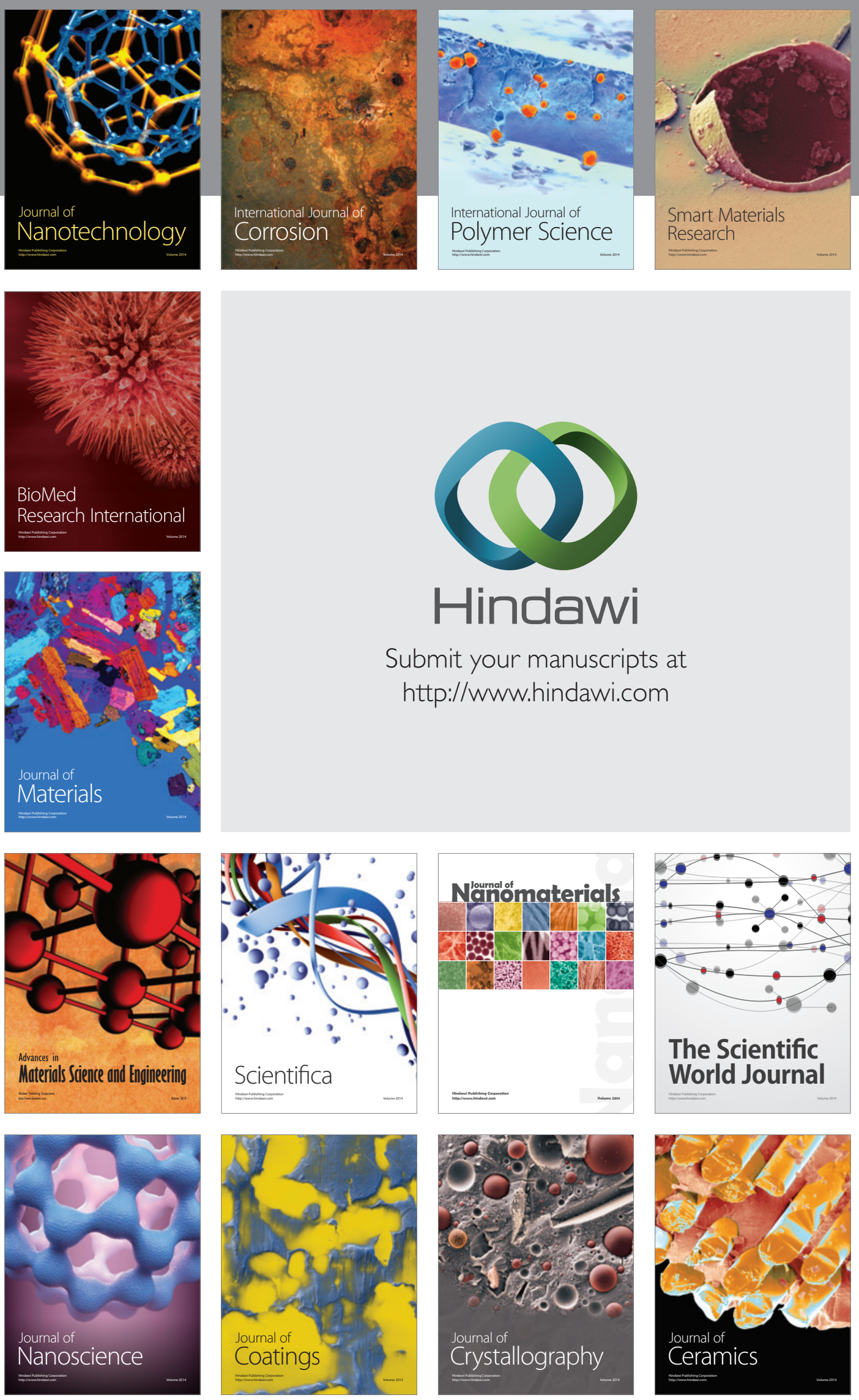

The Scientific World Journal

Submit your manuscripts at

http://www.hindawi.com

\section{World Journal}

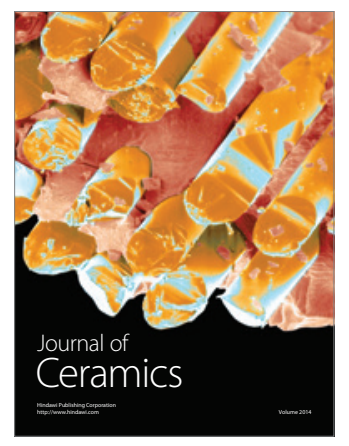

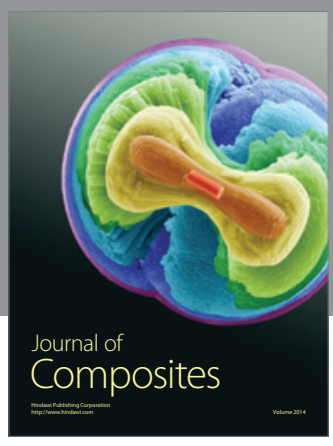
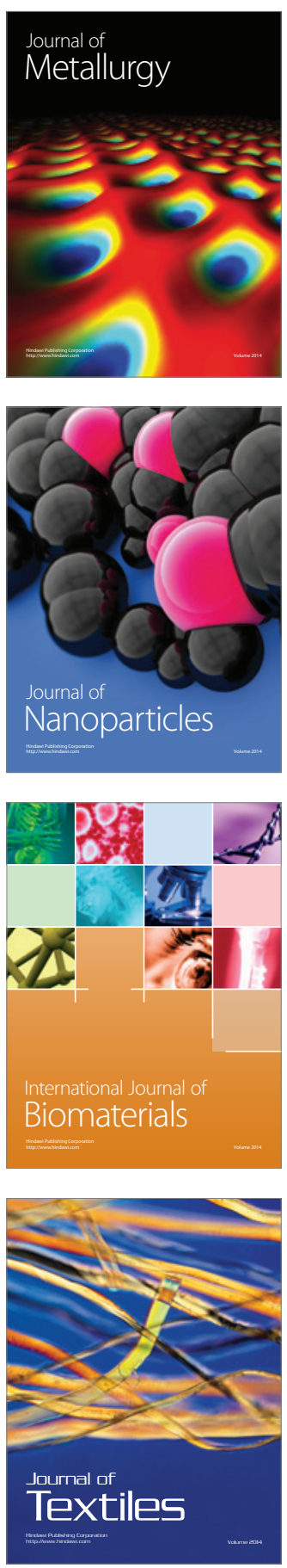